\title{
Demonstration of FoodFab: Creating Food Perceptual Illusions using Food 3D Printing
}

\author{
Parinya Punpongsanon \\ Osaka University \\ Toyonaka, Osaka, Japan \\ parinya@sys.es.osaka-u.ac.jp \\ Ying-Ju Lin \\ National Chiao Tung University \\ Hsinchu City, Taiwan \\ Xin Wen \\ MIT CSAIL \\ Cambridge, MA 02139, USA \\ xinwen@mit.edu \\ Kosuke Sato \\ Osaka University \\ Toyonaka, Osaka, Japan \\ sato@sys.es.osaka-u.ac.jp \\ Marianna Obrist \\ University of Sussex \\ Brighton, UK \\ m.obrist@sussex.ac.uk \\ Stefanie Mueller \\ MIT CSAIL \\ Cambridge, MA 02139, USA \\ stefanie.mueller@mit.edu \\ Daisuke Iwai \\ Osaka University \\ Toyonaka, Osaka, Japan \\ daisuke.iwai@sys.es.osaka- \\ u.ac.jp \\ Permission to make digital or hard copies of part or all of this work for personal or \\ classroom use is granted without fee provided that copies are not made or distributed \\ for profit or commercial advantage and that copies bear this notice and the full citation \\ on the first page. Copyrights for third-party components of this work must be honored \\ For all other uses, contact the owner/author(s). \\ Copyright held by the owner/author(s). \\ CHI'20, April 25-30, 2020, Honolulu, HI, USA \\ ACM 978-1-4503-6819-3/20/04. \\ https://doi.org/10.1145/3334480.XXXXXXX
}

\begin{abstract}
Food 3D printing enables the creation of customized food structures based on a person's individual needs. In this paper, we demonstrate the use of food 3D printing to create perceptual illusions for controlling the level of perceived satiety given a defined amount of calories. We present FoodFab, a system that allows users to control their food intake through modifying a food's internal structure via two $3 \mathrm{D}$ printing parameters: infill pattern and infill density. In two experiments with a total of 30 participants, we studied the effect of these parameters on users' chewing time that is known to affect people's feeling of satiety. Our results show that we can indeed modify the chewing time by varying infill pattern and density, and thus control perceived satiety. Based on the results, we propose two computational models and integrate them into a user interface that simplifies the creation of personalized food structures.
\end{abstract}

\section{Author Keywords}

personal fabrication; food perception; food-interaction design; food 3D printer; fabrication techniques

\section{CCS Concepts}

-Human-centered computing $\rightarrow$ Human computer interaction $(\mathrm{HCl})$; Interaction design; 


\section{Introduction}

Recently, food perception researchers have started to investigate how to change people's perception of satiety by modifying perceptual cues. Visual cues, for instance, play an important role: When a piece of food is cut into strips rather than cubes, people perceive it as more filling since it takes up more volume on the plate [3]. Similarly, haptic cues, such as an increased biting force and the resulting longer chewing time affect people's feeling of satiety [4]

Within $\mathrm{HCl}$, we see a growing effort to implement such perceptual cues digitally by overlaying content onto the food using augmented reality [2]. While augmented reality delivers effective illusions, it requires users to wear extra hardware and is less practical when used at home since users see the plain food before it is augmented.

With the proliferation of food 3D printing technology, the creation of computationally-controlled food perception illusions is no longer limited to the digital realm but can be tied back to the physical modification of food, which was originally used in food perception research.

\section{FoodFab: 3D Printed Food Illusions}

FoodFab is a food 3D printing system that prints food which induces different levels of satiety given a specified amount of calories. It accomplishes this by modifying the chewing time of the 3D printed food, which has been shown to correlate with satiety. FoodFab creates food structures of specific chewing times by varying two 3D printing parameters: the infill pattern and the infill density.

We chose to modify infill for two reasons: (1) variations in infill allow to vary the mechanical strength of the 3D printed food structures, which causes the changes in chewing pattern and time, and (2) variations in infill are not visible from the outside, i.e. hidden from the user.

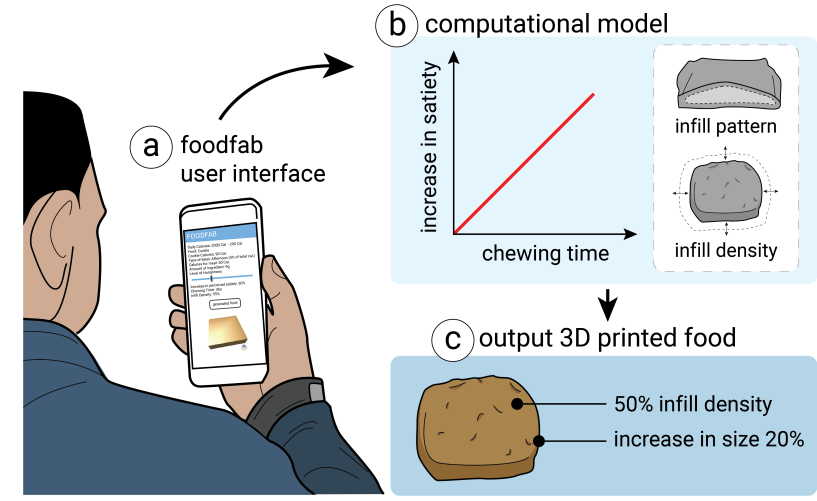

Figure 1: FoodFab is a system that controls a person's perceived satiety given a defined amount of calorie intake. It accomplishes this by creating food structures of different chewing times, which can be accomplished by varying 3D printing parameters, such as infill pattern and infill density. (a) Users input the type of food and level of hungriness, (b) FoodFab retrieves the required chewing time and matching infill parameters, and (c) 3D prints the food.

Infill Pattern: Infill patterns, such as honeycomb, rectilinear, and Hilbert, change how the infill path is laid out (Figure 4). The type of infill pattern affects the chewing time but does not affect the calorie amount since the path of each infill pattern is approximately the same length and thus requires approximately the same amount of ingredient.

Infill Density: Infill density defines how sparse the model is on its interior (Figure 3). The density of infill affects the chewing time since sparser structures have less mechanical strength. Since lower infill densities require less ingredient, the model is enlarged to keep the calorie amount constant for different densities.

To create a piece of food that induces a desired satiety level given a defined calorie amount, the FoodFab system takes 


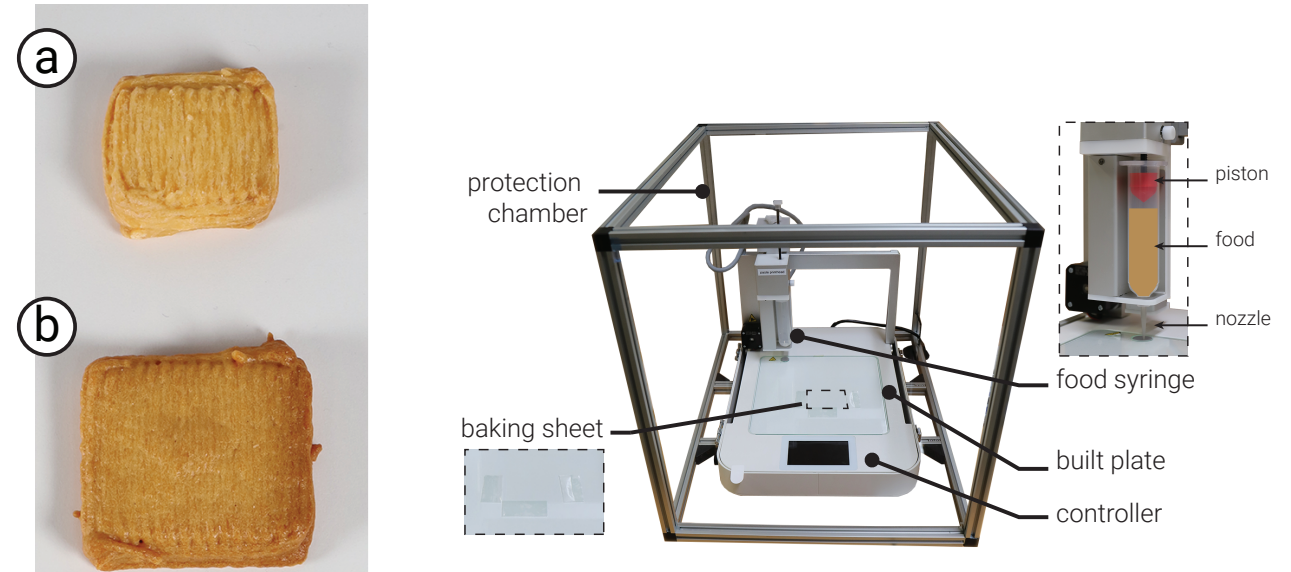

(C)

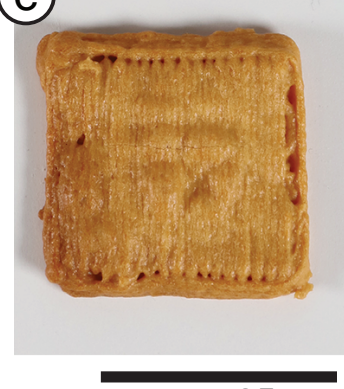

$25 \mathrm{~mm}$

Figure 3: Varying infill density, which also varies size: (a) $70 \%$ infill $(25 \times 25 \mathrm{~mm}),(\mathrm{b}) 55 \%$ infill $(35 \times 35 \times 5 \mathrm{~mm})$ and (c) $39 \%$ infill $(40 \times 40 \mathrm{~mm})$. All cookies are printed with $5 \mathrm{~mm}$ height.

Figure 2: Food 3D Printer 3DbyFlow used for fabricating the food structures. The baking sheet can be used to transfer the 3D printed food structure to an oven or pan. The protection chamber keeps the area clean and prevents airflow which may dry out the

as input a user's selection from a list of foods, a level of hunger, and the remaining calories for the day (e.g., from a user's fitness tracker). The system then outputs a set of $3 \mathrm{D}$ printing parameters and a 3D printable .gcode file that creates the a food structure of the desired chewing time.

\section{D Printing Hardware and 3D Printed Food}

All food structures printed were fabricated on the food 3D printer called 3DbyFlow that is shown in Figure 2. The 3 DbyFlow printer allows accurate printing and fabrication of food with different internal structures, necessary to deploy different infill patterns and densities. Based on our ex-

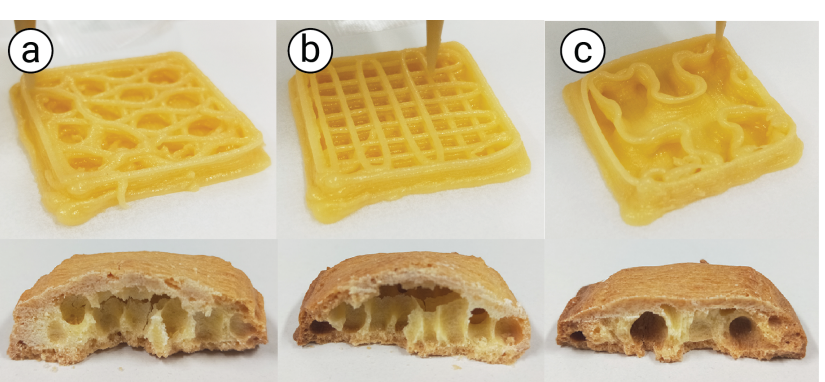
food while it is printing. perience with the 3D printer, food structures printed from the same 3D model (i.e., printed from the same ingredient amount) have an error rate of $\pm 0.10 \mathrm{~g}$, which we determined by weighting the 3D printed food structure using a standard weight scale (SHIMADZU ELB300).

Using the 3DbyFlow printer, we tested different ingredients
Figure 4: Varying Infill Pattern: (a) Honeycomb infill, (b) Rectilinear infill, and (c) Hilbert infill.

for creating 3D printed food structures. Since our work relies on internal structures of a specific pattern and density, we found that ingredients with the following properties work best for our approach: (1) form a sturdy structure at room temperature, (2) if post-processing (cooking, baking) is required, the food keeps the same shape, size, and internal structure.

\section{Applications and Demonstration}

To showcase the possibilities of FoodFab, we illustrate a range of different application scenarios:

Integration with Daily Meal Preparation: Food 3D printers recipes linked on the manufacturer website where a user can buy the ingredients as prefilled food capsules in supermarkets. FoodFab can be integrated into this emerging ecosystem around food 3D printers by using the computational models to personalize the recipes offered to consumers to help them manage their food intake.

Decreasing Food Size Over Time: People who overeat often have an attraction to large portion sizes to which they get accustomed over time [3]. Thus, to a person that that emerge on the consumer market tend to offer a list of 
overeats, a regularly sized meal may seem small in comparison. A future avenue for FoodFab would be to investigate if small changes in meal size over time can get users accustomed to regularly sized portions. We will demonstrate that we can modify the size of a piece of food by changing the infill density while keeping calories constant.

In our demonstration, we will shows our food 3D printer and the fabricated "cookie" with various infill parameters similar to the one used in the experiments (Figure 4 and Figure 3 ). We will also demonstrate our user interface that integrates the computational models with food 3D printing.

\section{Conclusion}

We demonstrated how to use personal fabrication devices to create food perceptual illusion. Rather than digitally augmenting food, we showed how to use food 3D printing to physically integrate perceptual illusions. For more details regarding the user studies and computational models, please refer to [1].

\section{Acknowledgements}

This work has been partially supported by the JSPS KAKENHI under Grant No. JP15H05925 and JP19K20321.

\section{REFERENCES}

[1] Ying-Ju Lin, Parinya Punpongsanon, Xin Wen, Daisuke Iwai, Kosuke Sato, Marianna Obrist, and
Stefanie Muller. 2020. FoodFab: Creating Food Perception Illusions using Food 3D Printing. In Proceedings of the $2020 \mathrm{CHI}$ Conference on Human Factors in Computing Systems (CHI '20). ACM, New York, NY, USA, 1-1. DOI :

http://dx.doi.org/10.1145/3313831.3376421

[2] Takuji Narumi, Yuki Ban, Takashi Kajinami, Tomohiro Tanikawa, and Michitaka Hirose. 2012. Augmented Perception of Satiety: Controlling Food Consumption by Changing Apparent Size of Food with Augmented Reality. In Proceedings of the SIGCHI Conference on Human Factors in Computing Systems (CHI '12). ACM, New York, NY, USA, 109-118. DOI : http://dx.doi.org/10.1145/2207676.2207693

[3] Sho Sakurai, Takuji Narumi, Yuki Ban, Tomohiro Tanikawa, and Michitaka Hirose. 2013. Affecting Our Perception of Satiety by Changing the Size of Virtual Dishes Displayed with a Tabletop Display. In Virtual, Augmented and Mixed Reality. Systems and Applications. Springer, 90-99.

[4] Nicolien Zijlstra, René de Wijk, Monica Mars, Annette Stafleu, and Cees de Graaf. 2009. Effect of bite size and oral processing time of a semisolid food on satiation. The American Journal of Clinical Nutrition 90, 2 (06 2009), 269-275. DOI :

http://dx.doi.org/10.3945/ajcn.2009.27694 